\title{
TURBO-CODED PARALLEL MODEM TECHNIQUES FOR PERSONAL COMMUNICATIONS
}

\author{
T. Keller, J.P Woodard and L. Hanzo \\ Dept. of Electr. and Comp. Sc., Univ. of Southampton, SO17 1BJ, UK. \\ Tel: +4417035931 25, Fax: +441703594508 \\ Email: tk, jpw, lh@ecs.soton.ac.uk \\ http://www-mobile.ecs.soton.ac.uk
}

\begin{abstract}
In this paper we study the performance of the wideband Orthogonal Frequency Division Multiplexing (OFDM) system in conjunction with channel coding based on turbo codes over a range of wideband Rayleigh fading channels. Due to their diversity effect wideband propagation channels provide similar gains for OFDM modems to those of equalised narrowband channels, resulting in substantial coding gains, when combined with turbo coding.
\end{abstract}

\section{INTRODUCTION}

The European Research in Communications Equipment (RACE) project endeavoured to comparatively study Time Division Multiple Access (TDMA) and Code Division Multiple Access (CDMA) for the third generation Universal Mobile Telecommunications System (UMTS). Many sophisticated techniques have been proposed and the Adavanced TDMA (ATDMA) framework emerged as an attractive candidate for supporting a variety of services, requiring different bitrates [1,2]. Although the ATDMA proposal gives cognizance to the different expected delay-spread constraints imposed by the various propagation environments, it is inevitable that in some cases the prevailing dispersion will exceed the expected values, which results in serious Bit Error Rate (BER) degradation in conventional serial modems, if no equaliser is used. The European FRAMES consortium is advocating a hybrid air-interface for their UMTS proposal [3], which contains the so-called Orthogonal Frequency Division Multiplex (OFDM) system [4] in its toolbox. Hence, as an attractive design alternative, it is beneficial to study the applicability of OFDM for the emerging UMTS.

Hence in this paper we study the combination of an OFDM system with channel coding based on turbo codes. In Section 2 we briefly describe the OFDM

VTC'97, 5-7. May, 1997,Phoenix, USA scheme, while in Section 3 we demonstrate how different channel models affect the fading characteristics experienced at the receiver. In Section 4 turbo codes are introduced before providing system performance figures in Section 5 and concluding in Section 6.

\section{OFDM MODEM}

Orthogonal Frequency Division Multiplexing (OFDM) is a special case of Code Division Multiplexing, using a set of $N$ mutually orthogonal signals $c_{n}(t)$ which are modulated individually to transmit $N$ data symbols $a_{n}$ of the data rate $1 / T_{a}$ in parallel over a period of time $N \cdot T_{s}$. OFDM was originally proposed by Chang [5], and is portrayed in more depth in [6] and [4].

In OFDM, the orthogonal signals are complex exponential functions of the form $c_{n}(t)=e^{j \omega_{n} t}$, where $\omega_{n}=$ $2 \pi\left(f_{0}+n \Delta f\right)$ and $\Delta f=1 /\left(N T_{s}\right)$. The resulting signal transmitted over the channel is $x(t)=\sum_{n=0}^{N-1} a_{n} \cdot c_{n}(t)$, which corresponds to a bundle of $N$ parallel subcarriers, each of which occupies $1 / N$ of the total signal spectrum. To combat inter-symbol interference, a guard interval containing the cyclic extension of the following OFDM symbol is inserted between consecutive OFDM symbols in the time domain.
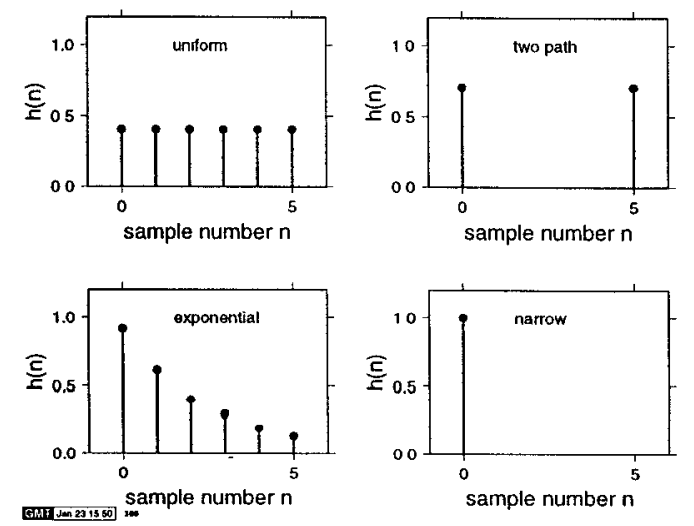

Figure 1: Impulse responses of the simulated wideband channels. 


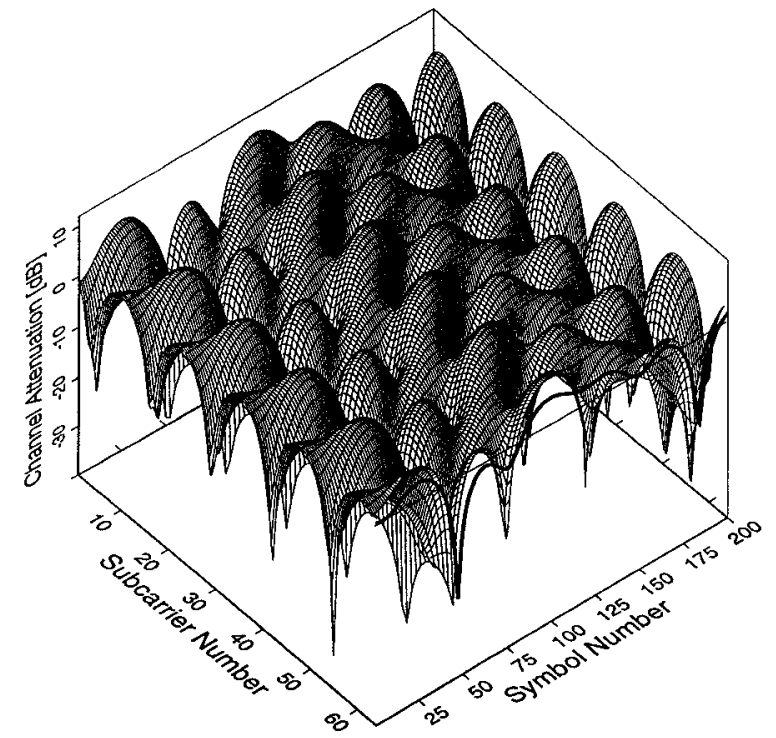

GMT $\operatorname{dan} 2216: 40,3091$

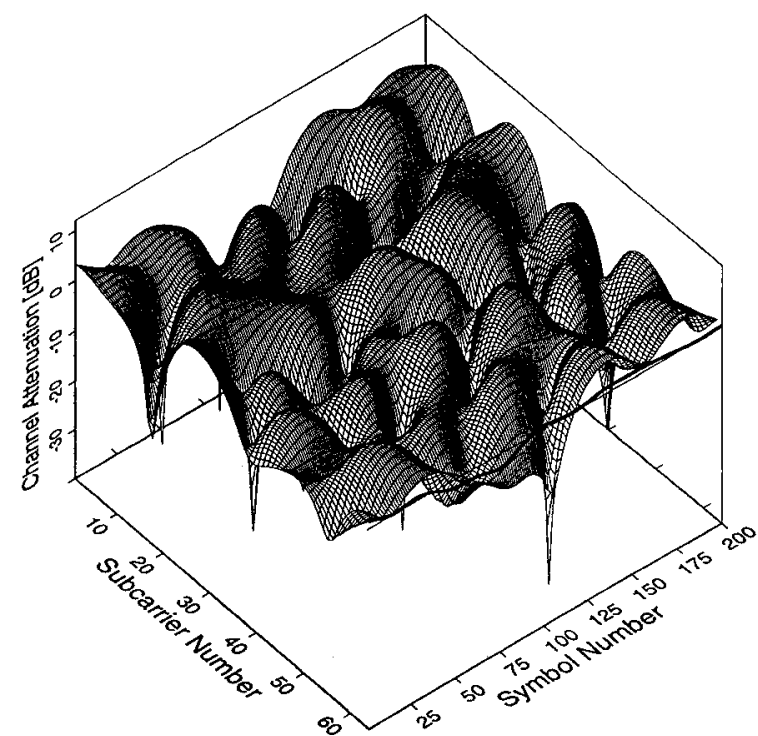

GMil Jan 22 18:35: 304

(a) two path model

(b) uniform six path model

Figure 2: Frequency selective channel amplitude for the two-path and uniform six-path models for 200 OFDM symbols with 64 subcarriers. The bold line above the symbol-index axis gives the average received subcarrier power per OFDM symbol.

OFDM systems are generally implemented using the Discrete Fourier Transform (DFT), mostly in the computationally more efficient Fast Fourier Transform (FFT) implementation. The time discrete output of the DFT is denoted $x(k)$.

The system assumed in this paper is a 64 subcarrier OFDM modem, employing a guard interval of 10 samples. No oversampling and pulse shaping is assumed, so that the transmitter emits time domain samples $x(k)$ at the rate $1 / T_{s}$. The wideband channel is modelled by a six tap FIR filter, whose time variant impulse response $h(n, t)$ is convolved with the transmitted time domain samples. White Gaussian noise $n(t)$ is added and the resulting sample sequence $y(k)=$ $x(k) * h(n, t)+n(t)$ is demultiplexed by the receiver FFT operation after the removal of the guard samples. Perfectly coherent detection is assumed in the frequency domain.

\section{CHANNEL MODEL}

The impulse responses $h(n)$ of the wide band radio channels examined consist of six impulses, spaced by the the channel symbol duration $T_{s}$. Three different wide band power delay profiles were investigated: a uniform, an exponentially decaying and a two path profile with

$$
h(n)=\left\{\begin{array}{lll}
1 / \sqrt{6} & n=0 \ldots 5 & \text { (uniform) } \\
A \cdot e^{B n} & n=0 \ldots 5 & \text { (exponential) } \\
1 / \sqrt{2} & n=0,5 & \text { (two path) }
\end{array}\right.
$$

where

$$
\begin{aligned}
B & =\frac{\ln 0.1}{5} \text { and } \\
\frac{1}{A} & =\left(\sum_{n=0}^{5} e^{2 B n}\right)^{1 / 2}
\end{aligned}
$$

The impulse responses are depicted in Figure 1.

All the paths in the impulse responses were treated as independent Rayleigh fading narrow band channels, with a normalised Doppler frequency of $2.44 \cdot 10^{-4}$, corresponding to a RACE ATDMA long macro cell type system with a carrier frequency of $1.9 \mathrm{GHz}$, a symbol rate of $360 \mathrm{kBaud}$ and a vehicular velocity of $50 \mathrm{~km} / \mathrm{h}$.

The convolution of the transmitted time domain signal with the channel impulse response results in frequency selective complex fading of the data symbols in the frequency domain. Since we assume perfectly coherent detection in the frequency domain in this contribution, only the amplitude of the frequency selective fading influences the reception of the data symbols. Figures 2(a) and 2(b) show the fading amplitude for the 


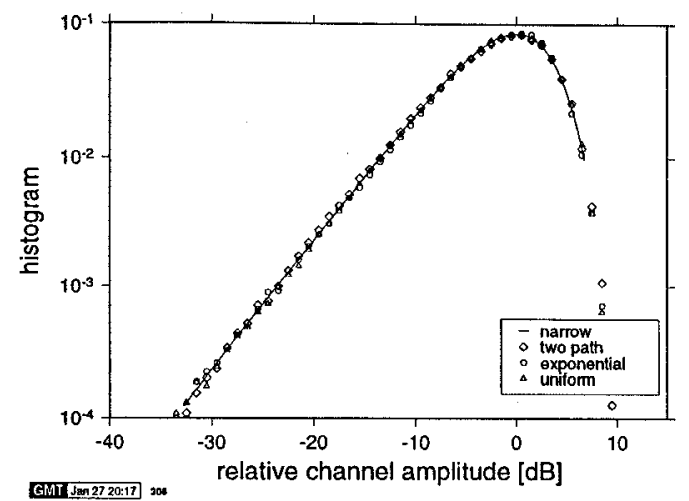

Figure 3: Histogram of relative frequency domain channel amplitude for narrowband, two path, exponential and uniform wideband channels.

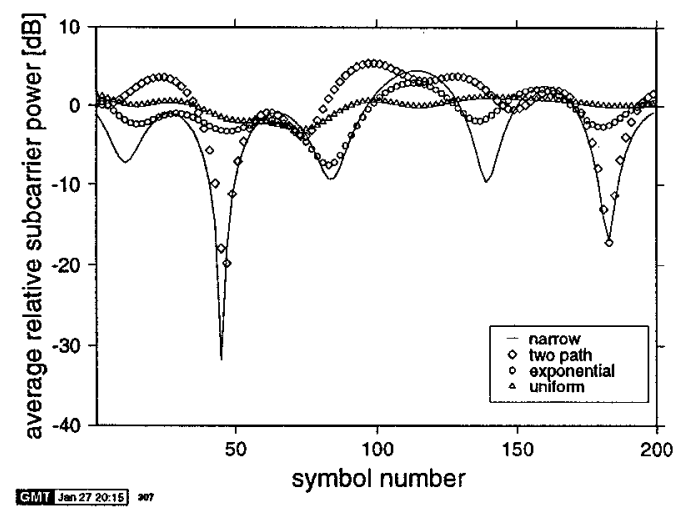

Figure 4: Average relative subcarrier power fluctuation for 200 consecutive OFDM symbols for narrowband as well as two path-, exponential- and uniform wideband channels

2-path and the uniform 6-path impulse responses over the system's frequency range of 64 subcarriers for 200 consecutive OFDM symbols on logarithmic $\mathrm{dB}$ scale. It is clear from these Figures that the channel characteristics vary with time, but the normalised Doppler frequency of the single paths is low compared to the OFDM symbol rate and hence the fading attenuation of consecutive OFDM symbols exhibits high correlation.

If perfect synchronisation is assumed, then the subcarriers are orthogonal, and no inter-subcarrier interference is present. We found that each OFDM subchannel symbol in the frequency domain experiences a narrowband fading channel. The experimental amplitude histogram of this equivalent frequency domain narrow band channel evaluated for all subchannels is given in Figure 3 for all the wide band channels considered along with the theoretical narrowband Rayleigh distribution. It can be seen that all the histograms follow the theoretical Rayleigh distribution indicated by the solid line. Since the time-domain channel impulse response taps are multiplied by the individual time-domain narrowband Rayleigh distributed fading envelopes, in the frequency-domain the corresponding spectra are convolved with eachother. Therefore the spectrum of the resultant wideband channel is given by the convolution of the frequency-domain transfer function corresponding to the channel's impulse response with the Doppler spectrum of the original narrowband Rayleigh fading channel.

In order to better understand the behaviour of the channel models studied and to allow us to further improve the system performance using forward error correction coding (FEC) we then studied the fluctuation of the average received subcarrier power per OFDM symbol as a function of the OFDM symbol-index, ie time, which is a simple measure of the diversity gain due to the multiple independently fading propagation paths. The bold line above the symbol index axis in Figure 2 demonstrates, how the average power per subcarrier for each OFDM symbol fluctuates as a function of the OFDM symbol index, ie time. It can be seen in the Figure that during the short observation period of this graph, the average power fluctuates much less in the uniform 6-path than in the 2-path case. The average received subcarrier power per OFDM symbol curves for the simulated wideband and the narrowband channels are plotted in Figure 4 as a function of the OFDM symbol index. Histograms of this average power fluctuation are shown for $10^{4}$ OFDM symbols in Figure 5. Clearly, the variations in the average power are largest for the narrow band Rayleigh fading channel, while the presence of more paths in the uniform 6-path model reduces the probability of very low received OFDM symbol powers.

\section{TURBO CODING}

Turbo coding is a relatively new form of channel coding, which was reported to approach the Shannonian performance predictions, when using a sufficiently high number of decoding iterations [7], as we will highlight shortly. The information sequence is encoded twice, with an interleaver between the inputs of the two encoders, in order to make the two encoded data sequences approximately statistically independent of each other. Quite often half rate Recursive Systematic Convolutional (RSC) encoders are used, with each RSC encoder producing a systematic output, which is equivalent to the original information sequence, as well as a stream of parity information. The two parity sequences are then punctured before being transmitted along with the original information sequence to the decoder. This puncturing of the parity information allows a wide range of coding rates to be realised, and often half the parity information from each encoder is sent. Along with the original data sequence this results in an overall coding rate of $1 / 2$. 

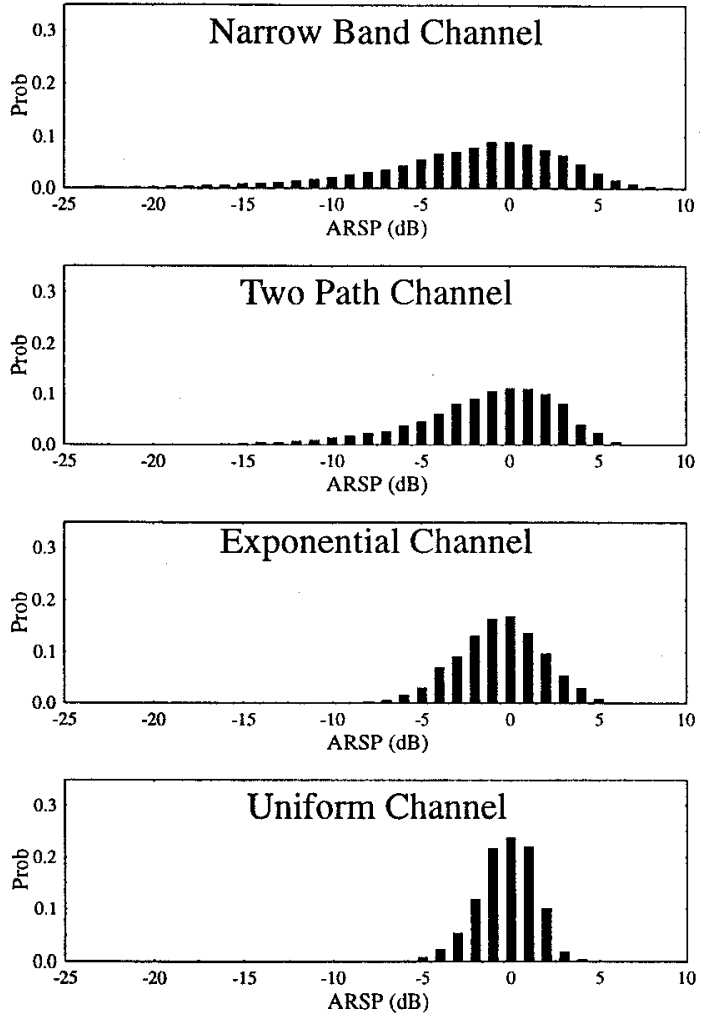

Figure 5: PDF of Average Received Subcarrier Power (ARSP) for Different Channel Models

At the decoder two RSC decoders are used. Special decoding algorithms must be used, which accept soft inputs and give soft outputs for the decoded sequence. These soft inputs and outputs provide not only an indication of whether a particular bit was a logical 0 or a 1 , but also a so-called likelihood ratio, which quantifies the probability that the bit has been correctly decoded.

The turbo decoder operates iteratively [7]. For every iteration each decoder uses the soft channel inputs, along with previously determined soft outputs from the other decoder, in order to determine a soft output giving the most likely decoded bit sequence. After each iteration the Bit Error Rate (BER) in the decoded sequence drops, but the improvements obtained with each iteration fall as the number of iterations increases. Hence for complexity reasons usually only 8 or 16 iterations are used.

As stated above, a turbo decoder uses two decoders which must both use soft inputs for the decoding process, and provide soft outputs giving an estimate of the decoded sequence. The Maximum A Posteriori (MAP) algorithm [8] is optimum for this task, but it is enormously complex. In our work we have used a modified version of the Viterbi algorithm, called the Soft Output Viterbi Algorithm (SOVA) [9], which is much less complex than the MAP algorithm. An $8 \times 8$-bit rectangular interleaver was used over one OFDM symbol. Hav-

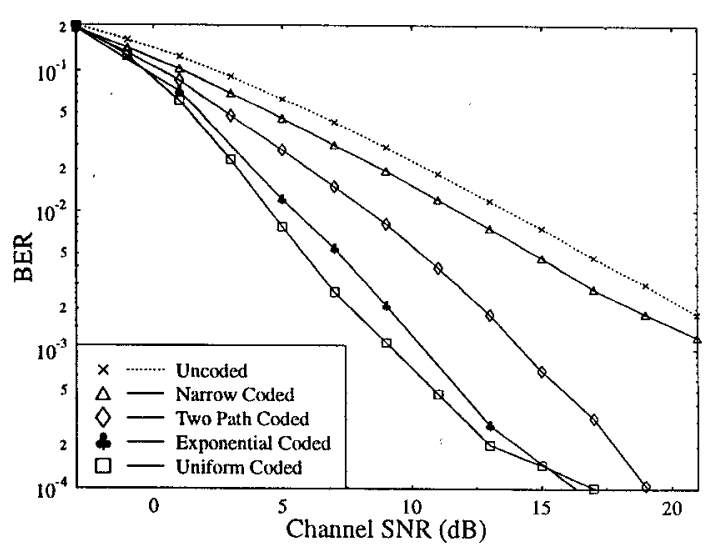

Figure 6: Bit Error Rate Performance of Coded and Uncoded Modulation over Different OFDM Channels

ing described the channel characteristics, the OFDM modem and the turbo coding schemes used let us now focus our attention on the system performance.

\section{SYSTEM PERFORMANCE}

The system components were simulated and the associated system performance curves are plotted in Figure 6 . Specifically, the performance of our OFDM scheme using simple BPSK modulation is shown by the dotted line in Figure 6. The uncoded BER is identical for all four channel models, since all four inflict fading amplitudes with a Rayleigh distribution, as shown in Figure 3.

As regards to FEC coding, we used a turbo coding scheme with two constraint length 2 RSC codes. A frame length of 64 information bits, with 8 decoding iterations, was employed. Figure 6 shows the Bit Error Rate (BER) performance of this channel coding scheme in conjunction with our BPSK modem over the four different wideband channels described earlier. Although all four channels give identical uncoded performance, it is clear that the BER is very dependent on the channel model, when channel coding is employed. This is due to their different variations in the Average Received Subcarrier Power (ARSP) for each OFDM symbol. As it was demonstrated by Figures 4 and 5, for the narrowband channel the ARSP fading attenuation can be quite high. When this happens, the channel outputs fed to the turbo decoder will be very unreliable, since they are swamped by noise. Hence the error correction power of the FEC code will be overloaded and ineffective. Consequently, the entire block of 64 coded data bits will suffer from a high BER. On the other hand, some OFDM symbols will have a high ARSP and the corresponding blocks of channel inputs to the turbo decoder will be er- 
ror free, and therefore again, the turbo decoder will be ineffective. Hence for a short-frame system with only 64 data bits sent per frame, turbo coding is ineffective over a narrowband Rayleigh channel, as seen in Figure 6.

The other channel models give ARSPs with progressively less variation from OFDM symbol to OFDM symbol for the exponential channel, the 2-path channel and the uniform 6-path channel. This in turn leads to progressively better BER performances, as can be seen from Figure 6. Figures 2(a) and 2(b) demonstrate that while in the former one occasionally a whole OFDM symbol is obliterated by fading, which is confirmed also by the ARSP-profiles plotted in bold, the 6-path channel is more immune to this phenomenon. For the uniform 6-path model it can be seen from Figure 5 in a different perspective that there is relatively little variation in the ARSP from OFDM symbol to OFDM symbol. Therefore, although the symbols associated with some subcarriers in an OFDM symbol may be corrupted, other subcarriers in the same OFDM symbol will be received reliably and hence the turbo decoder will not be swamped be the plethora of channel errors. Consequently the turbo decoder will be able to operate much more effectively than for the narrowband channel. This leads to a much higher coding gain for the uniform channel model than for the narrowband model, as can be seen from Figure 6 .

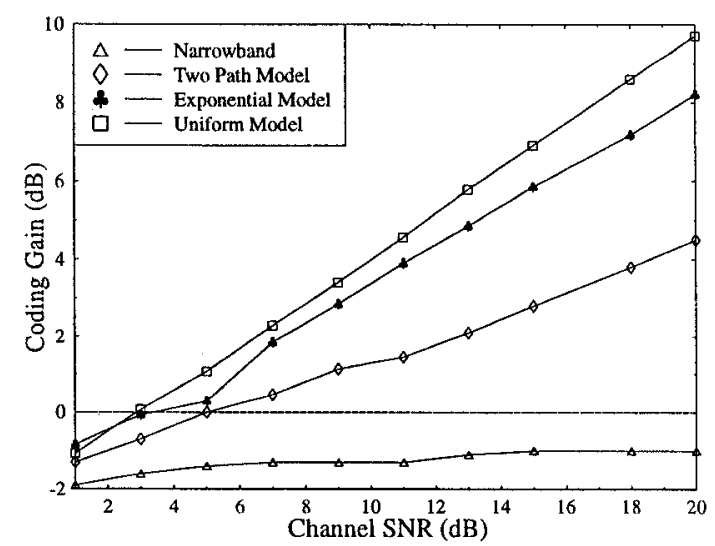

Figure 7: Coding gain for various channels

The corresponding coding gain plots are portrayed in Figures 7. These plots confirm it again that while for the narrowband channel the coding gain remains negative, for the wideband models a progressively improved coding gain is achieved for channel SNR values in excess of $3-4 \mathrm{~dB}$.

\section{CONCLUSIONS}

In this paper we have described an OFDM wideband system employing turbo codes. It has been shown that for a short-frame system with a narrowband Rayleigh channel, channel coding using turbo codes is relatively ineffective. However for wideband channels the OFDM system renders the Rayleigh channel relatively friendly and the turbo coding system then offers a substantial coding gain for channel SNR values in excess of 3-4 $\mathrm{dB}$.

\section{ACKNOWLEDGEMENT}

The financial support of Motorola ECID, Swindon, UK and that of the European Community in the framework of the FIRST and MEDIAN projects as well as that of the Physical Sciences Research Council is gratefully acknowledged. The authors are also indebted to the FIRST and MEDIAN consortia as well as to Jeff Torrance and Marco Breiling for stimulating discussions on the topic.

\section{REFERENCES}

[1] A. Urie, M. Streeton, "An advanced TDMA mobile access system for UMTS," IEEE Communications Magazine, pp. 38-47, Feb 1995.

[2] "Mobile communication networks, general aspects and evolution." European RACE D731 Public Deliverable, Sept 1995

[3] T. Ojanpera et al: Comparative study of hybrid multiple access schemes for UMTS, Proceedings of ACTS Summit, Granada, Spain, Nov. 27-29, 1996, pp 124-130

[4] W.T. Webb, L. Hanzo, Modern quadrature amplitude modulation: Principles and applications for fixed and wireless channels. IEEE Press-Pentech Press, 1994.

[5] R.W. Chang, "Synthesis of Band-Limited Orthogonal Signals for Multichannel Data Transmission," BSTJ, Dec 1966.

[6] Leonard J. Cimini, "Analysis and Simulation of a Digital Mobile Channel Using Orthogonal Frequency Division Multiplexing," IEEE Transactions on Communications, pp. 665-675, July 1985.

[7] Clande Berrou, Alain Glavieux and Punya Thitimajshima, "Near Shannon Limit Error-Corrcting Coding and Decoding: Turbo Codes," Proceedings of the International Conference on Communications, pp. 10641070, May 1993

[8] L.R. Bahl, J. Cocke, F. Jelinek and J. Raviv, "Optimal Decoding of Linear Codes for Minimising Symbol Error Rate," IEEE Transactions on Information Theory, pp. 284-287, March 1974.

[9] J. Hagenauer and P. Hoeher, "A Viterbi Algorithm with Soft-Decision Outputs and its Applications," IEEE Globecom, pp. 1680-1686, 1989. 